\title{
A Modal Logic on Judgment Aggregation
}

\author{
Li Dai ${ }^{1}$, Zhong Lei $^{2}$, Jihua Zhang ${ }^{3}$ \\ 1) School of Literature and Chinese as a Foreign Language, Mianyang Normal University, Mianyang, Sichuan, China \\ (daili227@163.com) \\ 2) Academic Affairs, Mianyang Normal University, Mianyang, Sichuan, China (leizhong8823@ 163.com) \\ 3) Department of Science and Technology, Mianyang Normal University, Mianyang, Sichuan, China (bzhangjihuajyu@126.com) \\ Abstract-Preference aggregation has been deeply studied in the social choice theory. Judgment aggregation becomes the \\ studying center of the social choice theory gradually. From the perspective of logic, judgment aggregation considers how to aggregate \\ many logical consistent formula sets into one logical consistent formula set. On the basis of the judgment aggregation modal logic $J A L$ \\ analyzed, we construct a preference aggregation logic system $J A L\left(L^{K}\right)$ using the language of the first-order logic. Thus the preference \\ aggregation questions could be converted into the judgment aggregation questions to be handled, so that the generality of the judgment \\ aggregation model is highlighted.
}

Keywords_-Rationality, Preference aggregation, Judgment aggregation, Modal logic

\section{一个判断聚合的模态逻辑}

\author{
代利 ${ }^{1}$ 雷忠 ${ }^{2}$ 张继华 ${ }^{3}$ \\ 1) 绵阳师范学院文学与对外汉语学院, 绵阳, 四川, 中国 \\ 2) 绵阳师范学院教务处, 绵阳, 四川, 中国 \\ 3) 绵阳师范学院科技处, 绵阳, 四川, 中国
}

\begin{abstract}
摘 要 偏好聚合在社会选择理论中已得到深入研究, 而近年出现的判断聚合逐渐成为社会选择理论的研究中心。判断聚合是从 逻辑的视角更一般地研究聚合, 探讨如何将多个一致的逻辑公式集聚合成一个一致的逻辑公式集。我们重点分析了一个判断聚合的模 态逻辑 $J A L$, 利用一阶逻辑的语言基于 $J A L$ 可以构建一个偏好聚合的逻辑系统 $J A L\left(\mathrm{~L}^{K}\right)$, 从而可将偏好聚合转化为判断聚合来处理, 突显了判断聚合模型的一般性。
\end{abstract}

关键词 理性, 偏好聚合, 判断聚合, 模态逻辑

\section{1. 引言}

如何将个体理性聚合为群体理性是社会理性认知研究 面临的主要难题, 同时也是经济学、哲学、政治学、法学 及计算机科学等多学科交叉研究的热点问题之一。美国著 名经济学家阿罗(Kenneth Arrow)教授以公理化的方法刻画 了个体理性偏好与群体理性偏好, 在此基础上证明了将理 性个体偏好聚合为理性群体偏好的不可能性。如何理解刻 画这种不可能性以期化解, 寻求合理的群体理性聚合方法

本文受四川省教育厅科研项目“现代逻辑视域下的群体理性聚合 研究”(13SB0159)及绵阳师范学院引进人才科研基金资助项目“基 于判断聚合逻辑的群体理性条件研究”(QD2012B03)资助。
成为相关研究亟待突破的理论瓶颈, 近年出现的判断聚合 研究为这一问题的突破提供了新思路。

\section{2. 偏好聚合与判断聚合}

偏好聚合关注的中心是将一个备选方案集合上多个主 体的偏好关系聚合成代表这个集体偏好的一个群体偏好关 系的社会福利函数, 判断聚合关注的中心是聚合逻辑公式 集的判断聚合规则。

\section{1 社会福利函数}

直观上讲, 一个社会福利函数是指这样一个过程或规 则: 以所有个体的偏好关系(一个偏好关系就是备选方案集 
上的一个特定的二元关系)为输入, 输出一个代表这个群体 偏好的偏好关系。为了讨论问题的方便, 我们假设下面所 讨论的偏好关系都是严格偏好关系。

从形式上说, 令 $K=\{a, b, c, \ldots\}$ 是一个备选方案的集合

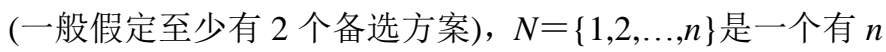
个主体的主体集(一般假定至少有 2 个主体)。 $K$ 上的一个偏 好关系 $R$ 是 $K$ 上的一个严格线性序, 即 $K$ 上的一个反对称 的(对于任意两个备选方案 $a$ 与 $b$, 如果 $a R b$, 那么并非 $b R a$ )、 传递的(对于任意三个备选方案 $a 、 b 、 c$, 如果 $a R b$ 并且 $b R c$, 那么 $a R c$ )、完全的(对于任意两个备选方案 $a$ 与 $b, a R b$ 或 者 $b R a)$ 二元关系。 $R_{i}$ 表示 $K$ 上主体 $i$ 的一个偏好关系, $L(K)$ 表示 $K$ 上所有偏好关系的集合。一个偏好组合就是一个 $n$ 元组 $\left(R_{1}, R_{2}, \ldots, R_{n}\right) \in L(K)^{n}$, 其中 $R_{i}(1 \leq i \leq n)$ 表示主体 $i$ 的一个偏 好关系。一个社会福利函数就是这样一个函数 $F$ : $L(K)^{n} \rightarrow L(K)$ 。

通常认为, 一个社会福利函数 $F$ 应该满足以下合理条 件:

(1) 无关选项独立性 (independence of irrelevant alternatives):

群体对于任意两个备选方案的偏好排序仅仅依赖于个 体们对这两个备选方案的偏好排序, 与其他因素无关。可 用公式表示为:

$\forall\left(R_{1}, \ldots, R_{n}\right) \in L(K)^{n} \forall\left(S_{1}, \ldots, S_{n}\right) \in L(K)^{n} \forall a \in K \forall b \in K(\forall i \in N$ $\left.\left(a R_{i} b \Leftrightarrow a S_{i} b\right) \Rightarrow\left(a F\left(R_{1}, \ldots, R_{n}\right) b \Leftrightarrow a F\left(S_{1}, \ldots, S_{n}\right) b\right)\right)$

(2)帕累托最优原则(Pareto optimality principle):

如果所有个体都认为一个备选方案优于另一个, 那么 群体也应同样如此认为。可用公式表示为:

$$
\begin{aligned}
& \forall\left(R_{1}, \ldots, R_{n}\right) \in L(K)^{n} \forall a \in K \forall b \in K\left(\forall i \in N a R _ { i } b \Rightarrow a F \left(R_{1}, \ldots\right.\right. \\
& \left.\left., R_{n}\right) b\right)
\end{aligned}
$$

(3)非独裁性(non-dictatorship):

不应使某个固定个体的偏好总是成为群体的偏好, 而 不管其他个体的偏好与之是如何不同。可用公式表示为:

$$
\neg \exists i \in N \forall\left(R_{1}, \ldots, R_{n}\right) \in L(K)^{n} F\left(R_{1}, \ldots, R_{n}\right)=R_{i}
$$

上述条件看上去是那样的自然而合情合理, 以致人们 通常把它们看作是社会福利函数应该满足的不言而喻的公 理。但是, 阿罗教授在《社会选择与个人价值》一书中证 明了: 如果备选方案不少于 3 个, 那么不存在同时满足无 关选项独立性、帕累托最优原则及非独裁性这三个显然合 理条件的社会福利函数。这就是著名的“阿罗不可能性定 理”, 也简称为“阿罗定理”。 ${ }^{[1]}$ 阿罗定理是社会选择理论中

[1] Kenneth J·Arrow, Individual Values and Social Choice. New York: Wiley, 1951; 2d ed., 1963.
一个里程碑式的结论, 阿罗定理揭示了: 把理性的个体偏 好聚合为理性群体偏好的不可能性。

\section{2 判断聚合规则}

判断聚合是从逻辑的视角来研究聚合, 判断聚合所关 注的问题是: 当每个主体都被给予一个代表他们判断或信 念的一致的逻辑公式集时, 如何通过所谓的判断聚合规则, 将这些逻辑公式集聚合成代表这个群体共同判断或信念的 一个一致的逻辑公式集。

从形式上说, 令 $N=\{1,2, \ldots, n\}$ 是一个有 $n$ 个主体的主 体集 (一般假定至少有 2 个主体), $\mathrm{L}$ 是我们所讨论的判断 聚合的一个基础逻辑, 其语言 $\mathscr{L}(\mathrm{L})$ 包含 $、$ 、 $\rightarrow$ 这两个具有 通常逻辑语义的联结词。基于 $\mathrm{L}$ 之上的一个议程 (agenda) 是一个非空集合 $A \subseteq \mathscr{L}(\mathrm{L})$, 对于任意一个不以 $\neg$ 开始的公式 $\Phi, \Phi \in A$ 当且仅当 $\neg \Phi \in A$, 这里假设双重否定可以相互抵 消, 即 $\neg \neg \Phi$ 就是 $\Phi$ 。通常把 $A$ 的一个元素称为是一个议项 (agenda item)。对于 $A$ 的任意一个子集 $A^{\prime}$, 如果不存在一 个公式 $\Phi \in \mathscr{L}(\mathrm{L})$, 使得 $A^{\prime} \vdash_{\mathrm{L}} \Phi \wedge \neg \Phi$, 那么 $A^{\prime}$ 是一致的。如 果对于每个不以 $\neg$ 开始的公式 $\Phi \in A, \Phi \in A^{\prime}$ 或者 $\neg \Phi \in A^{\prime}$, 那 么 $A^{\prime}$ 是完全的。一个主体 $i$ 的个体判断集是议程 $A$ 的一个 完全且一致的子集 $A_{i}, p \in A_{i}$ 表示主体 $i$ 赞同议项 $p$, 即主 体 $i$ 判断 $p$ 为真。一个判断组合是一个 $n$ 元组 $\left(A_{1}, A_{2}, \ldots, A_{n}\right)$, 其中 $A_{i}(1 \leq i \leq n)$ 是主体 $i(1 \leq i \leq n)$ 的个体判断集。J $(A, \mathrm{~L})$ 表示议 程 $A$ 上所有（个体或群体）判断集的集合, $J(A, \mathrm{~L})^{n}$ 表示议 程 $A$ 上所有判断组合的集合。如果 $\gamma \in J(A, \mathrm{~L})^{n}$, 用 $\gamma_{i}(1 \leq i \leq n)$ 表示 $\gamma$ 的第 $i$ 个元素 $\left(\gamma=\left(\gamma_{1}, \ldots, \gamma_{3}\right)\right)$, 即在判断组合 $\gamma$ 中主体 $i$ 的个体判断集。一个判断聚合规则就是这样一个函数 $f$ : $J(A, \mathrm{~L})^{n} \rightarrow J(A, \mathrm{~L})$ 。

一个判断聚合规则通常应该满足以下合理条件:

(1)独立性 (independence):

群体对于议程中任一议项的判断仅仅依赖于个体们对 于这个议项的判断, 与其他因素无关。可用公式表示为:

$$
\begin{aligned}
\forall p \in A \forall\left(A_{1}, \ldots, A_{n}\right) \in J(A, \mathrm{~L})^{n} \forall\left(B_{1}, \ldots, B_{n}\right) \in J(A, \mathrm{~L})^{n}(\forall i \in N(p \\
\left.\in A_{i} \Leftrightarrow p \in B_{i}\right) \Rightarrow\left(p \in f ( A _ { 1 } , \ldots , A _ { n } ) \Leftrightarrow p \in f \left(B_{\left.\left.\left.1, \ldots, B_{n}\right)\right)\right)}\right.\right.
\end{aligned}
$$

(2)统一性(unanimity):

如果所有个体都赞同议程中的某个议项, 那么群体也 应赞同这个议项。可用公式表示为:

$\forall p \in A \forall\left(A_{1}, \ldots, A_{n}\right) \in J(A, \mathrm{~L})^{n}\left(\forall i \in N p \in A_{i} \Rightarrow p \in f\left(A_{1}, \ldots, A_{n}\right)\right)$

(3)非独裁性(non-dictatorship):

不应使某个固定个体的判断总是成为群体判断, 而不 管其他个体的判断与之是如何的不同。可用公式表示为: 
$\neg \exists i \in N \forall\left(A_{1}, \ldots, A_{n}\right) \in J(A, \mathrm{~L})^{n} f\left(A_{1}, \ldots, A_{n}\right)=A_{i}$

判断困境 (Discursive Dilemma): 如果有至少三个主 体要对至少两个不同的公式 $p 、 q$ 以及它们的实质蕴涵式 $p \rightarrow q$ 做出判断, 那么运用关于命题的多数投票聚合规则可 能会得到一个不一致的群体判断。

我们将基于判断聚合逻辑 $J A L$, 探讨判断聚合与偏好 聚合之间的关系。

\section{3. 判断聚合的模态逻辑 $J A L$}

\section{$3.1 J A L$ 语形}

判断聚合逻辑 $J A L$ 的语言 $\mathcal{L}(N, A)$ 有两个参数, 其中 $N=\{1,2, \ldots, n\}$ 是一个有 $n$ 个主体的主体集， $A$ 是一个议程。 $J A L$ 的原子命题集为:

$$
\Pi=\{i, \sigma, \mathrm{h} p \mid i \in N, p \in A\}
$$

其中 $\sigma$ 是一个常量, 表示当前议项为群体所赞同。 $J A L$ 语言 $\mathcal{L}(N, A)$ 的(合式)公式递归定义如下:

$\Phi::=\alpha|\square \Phi| \square \Phi|\Phi \wedge \Phi| \neg \Phi \quad\left(\alpha \in \prod\right)$

口和 $\square$ 的对偶算子定义如下:

$\diamond \Phi:=\neg \square \neg \Phi$

$\diamond \Phi:=\neg \square \neg \Phi$

\section{$3.2 J A L$ 语义}

相对于 $\mathcal{L}(N, A)$ 及基础逻辑 $\mathrm{L}$ 的一个模型就是议程 $A$ 上 的一个判断聚合规则 $f$ 。 $\mathcal{L}(N, A)$ 在一个三元组 $\langle f, \gamma, p\rangle$ 上进行 解释, 一个三元组 $\langle f, \gamma, p>$ 被称为是一个表 (table), 其中 $f$ 是一个判断聚合规则, $\gamma$ 是一个判断组合, $p$ 是一个议项。

一个公式在一个表上的语义解释如下:

(1) $f, \gamma, p \vDash_{\mathrm{L}} \mathrm{h} q$ 当且仅当 $p=q$;

(2) $f, \gamma, p \vDash_{\mathrm{L}} i$ 当且仅当 $p \in \gamma_{i}$;

(3) $f, \gamma, p \vDash_{\mathrm{L}} \sigma$ 当且仅当 $p \in f(\gamma)$;

(4) $f, \gamma, p \vDash_{\mathrm{L}} \square \Psi$ 当且仅当 $\forall \gamma^{\prime} \in J(A, \mathrm{~L})^{n} f, \gamma^{\prime}, p \vDash_{\mathrm{L}} \Psi$;

(5) $f, \gamma, p \vDash_{\mathrm{L}} \boldsymbol{\square} \Psi$ 当且仅当 $\forall p^{\prime} \in A f, \gamma, p^{\prime} \vDash_{\mathrm{L}} \Psi$;

(6) $f, \gamma, p \vDash_{\mathrm{L}} \Phi \wedge \Psi$ 当且仅当 $f, \gamma, p \vDash_{\mathrm{L}} \Phi$ 并且 $f, \gamma, p \vDash_{\mathrm{L}} \Psi$;

(7) $f, \gamma, p \vDash_{\mathrm{L}} \neg \Phi$ 当且仅当 $f, \gamma, p \vDash_{\mathrm{L}} \Phi$ 。

直观上说, (1)的意思是“公式 $\mathrm{h} q$ 在表 $\langle f, \gamma, p\rangle$ 上为真, 当且仅当在运用判断聚合规则 $f$ 对判断组合 $\gamma$ 的聚合中, 当 下所考虑的议项是 $q$ ”; (2) 的意思是“公式 $i$ 在表 $\langle f, \gamma, p\rangle$ 上为 真, 当且仅当在运用判断聚合规则 $f$ 对判断组合 $\gamma$ 的聚合中, 议项 $p$ 是主体 $i$ 的个体判断集的元素”; (3)的意思是“公式 $\sigma$ 在表 $\langle f, \gamma, p\rangle$ 上为真, 当且仅当在运用判断聚合规则 $f$ 对判断
组合 $\gamma$ 的聚合中, 议项 $p$ 是聚合得到的群体判断集 $f(\gamma)$ 的元 素”; (4)的意思是“公式 $\square \Psi$ 在表 $\langle f, \gamma, p\rangle$ 上为真, 当且仅当对 于议项 $p$ 来说，在运用判断聚合规则 $f$ 对所有的判断组合 的聚合中, $\Psi$ 都是真的”; (5)的意思是“公式 $\Psi$ 在表 $\langle f, \gamma, p\rangle$ 上为真，当且仅当在运用判断聚合规则 $f$ 对判断组合 $\gamma$ 的聚 合中, 对于所有议项来说, $\Psi$ 都是真的”。命题联结词“冺

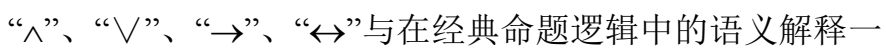
样。 $\diamond \Phi$ 表示“ $\Phi$ 在某个判断组合中是真的”; $\Phi$ 表示“ $\Phi$ 对 于某个议项来说是真的”。

令 $f$ 是一判断聚合规则, $\Phi$ 是一 $\mathcal{L}(N, A)$ 公式，如果对 于议程 $A$ 上任一判断组合 $\gamma$ 、任一议项 $p \in A$, 都有 $f, \gamma, p \vDash_{\mathrm{L}} \Phi$, 则 $\Phi$ 在 $f$ 中为真, 记作 $f \vDash_{\mathrm{L}} \Phi ; \vDash_{\mathrm{L}} \Phi$ 当且仅当对于任一 $f$, 都有 $f \vDash_{\mathrm{L}} \Phi$ 。一个输出 $o$ 是一个合取式 $k_{1} \wedge \ldots \wedge k_{n}$, 其中每一 个 $k_{i}(1 \leq i \leq n)$, 要么是 $i$, 要么是 $\neg i$ 。通常用 $O$ 表示所有可能 输出的集合。

如果一个公式在一个判断聚合规则 $f$ 中是真的当且仅 当 $f$ 具有某一性质, 那么我们说这个公式表达了这一性质。 判断聚合规则的一些性质可以用 $J A L$ 语言 $\mathcal{L}(N, A)$ 表达:

(1)独立性: $\square \wedge_{o \in O} \mathbf{\square}((o \wedge \sigma) \rightarrow \square(o \wedge \sigma))$;

(2)统一性: $\square \mathbf{\square}((1 \wedge \ldots \wedge n) \rightarrow \sigma)$;

(3)非独裁性: $\wedge_{i \in N} \diamond \neg(\sigma \leftrightarrow i)$ 。

给定基础逻辑 $\mathrm{L}$ 、主体集 $N$ 及 $\mathrm{L}$ 之上的一个有穷议程 $A$, 基于语言 $\mathcal{L}(N, A)$ 可以定义一个可靠且完全的判断聚合 逻辑 $J A L(\mathrm{~L})$ (当 $\mathrm{L}$ 明确时, 直接记为 $J A L)$, 具体可参见[2]。

\section{4. 基于 $J A L$ 的偏好聚合逻辑}

Dietrich,List已证明可以将偏好聚合嵌入到判断聚合之 中([6])。在这里, 我们将阐明判断聚合逻辑 $J A L$ 也可以用于 分析偏好聚合。当把偏好聚合视为判断聚合的一个特例时, 由判断聚合逻辑 $J A L$, 也可以得到一个偏好聚合的逻辑 $J A L\left(\mathrm{~L}^{K}\right)$ 。

给定一个有穷备选方案的集合 $K=\{a, b, c, \ldots, w\}$ (一般假 定至少有 2 个备选方案), 可定义一个简单的谓词逻辑 $\mathrm{L}^{K}$ 如 下 $^{[2]}$ :

- $\quad \mathrm{L}^{K}$ 的语言 $\mathcal{L}\left(\mathrm{L}^{K}\right)$ 具有如下符号: 用于表示备选方案的 集合 $K=\{a, b, c, \ldots, w\}$ 中的每一个备选方案的个体常元符 号 $a, b, c, \ldots, w$; 个体变元符号 $v_{1}, v_{2}, \ldots$; 一个二元等同谓 词符号 $=$; 用于表示严格偏好的一个二元谓词符号 $P$ 以 及通常的命题逻辑、经典谓词逻辑的联结词。

- $\mathrm{L}^{K}$ 还具有下列刻画理性偏好的公理:

[2] Dietrich, F., and List, C. (2007).Arrow's theorem in judgement aggregation. Social Choice and Welfare ,.29 (1),19-33. 


$$
\forall v_{1} \forall v_{2}\left(v_{1} P v_{2} \rightarrow \neg v_{2} P v_{1}\right)
$$

(对应了偏好关系的非对称性)

$\forall v_{1} \forall v_{2} \forall v_{3}\left(v_{1} P v_{2} \wedge v_{2} P v_{3} \rightarrow v_{1} P v_{3}\right)$

(对应了偏好关系的传递性)

$\forall v_{1} \forall v_{2}\left(\neg v_{1}=v_{2} \rightarrow\left(v_{1} P v_{2} \vee v_{2} P v_{1}\right)\right)$

(对应了偏好关系的完全性)

在 $K$ 上所有偏好关系 (完全的线性序) 的集合与偏好议 程 $A^{K}$ 上所有 $\mathrm{L}^{K}$-一致且完全的判断集的集合之间有一个一 一对应关系, 其中偏好议程 $A^{K}=\{a P b, \neg a P b: a, b \in K, a \neq b\}(a P b$ 表示命题 “ $a$ 比 $b$ 好” )。给定 $K$ 上的一个社会福利函数 $F$, 对应 的偏好议程 $A^{K}$ 上的判断聚合规则 $f^{F}$ 定义如下: $f^{F}\left(A_{1}, \ldots, A_{n}\right)=A$, 其中 $A$ 是与 $F\left(L_{1}, \ldots, L_{n}\right)$ 相对应的 $\mathrm{L}^{K}$-一致且完 全的群体判断集, $L_{i}$ 是与 $\mathrm{L}^{K}$-一致且完全的个体判断集 $A_{i}$ 相 对应的个体偏好关系。

这样, 我们就能利用 $J A L\left(\mathrm{~L}^{K}\right)$ 来分析偏好聚合了: 这里 的逻辑语言是 $\mathcal{L}\left(N, A^{K}\right)$, 其中 $N$ 为某一主体集, $\mathrm{L}^{K}$ 为基础逻 辑。在 $K$ 上的一个社会福利函数 $F$ 、一个偏好组合 $L \in L(K)$ 及一个有序对 $(a, b) \in K \times K(a \neq b)$ 中对公式 $\Phi$ 的解释定义如下:

$F, L,(a, b) \vDash{ }^{s w f} \Phi \Leftrightarrow f^{F}, \gamma^{L}, a P b \vDash_{\mathrm{L}}{ }^{K} \Phi$

其中 $\gamma^{L}$ 是与偏好组合 $L$ 相对应的判断组合。

令 $F$ 是一社会福利函数, $\Phi$ 是一 $\mathcal{L}\left(N, A^{K}\right)$ 公式, 如果 对于任一偏好组合 $L$ 、任一备选方案有序对 $(a, b)$, 都有 $F, L,(a, b) \vDash^{s w f} \Phi$, 则 $\Phi$ 在 $F$ 中为真, 记作 $F \vDash^{s w f} \Phi$ 。 $\vDash^{s w f} \Phi$ 当 且仅当对于任一 $F$,都有 $F F^{s w f} \Phi$ 。

如果一个公式在一个社会福利函数 $F$ 中是真的当且仅 当 $F$ 具有某一性质, 那么我们说这个公式表达了这一性质。 社会福利函数的一些性质可以用 $J A L$ 语言 $\mathcal{L}(N, A)$ 表达:

(1)无关选项独立性: $\square \wedge_{o \in O} \square((o \wedge \sigma) \rightarrow \square(o \wedge \sigma))$;

(2)帕累托最优原则: $\square$ - $((1 \wedge \ldots \wedge n) \rightarrow \sigma)$;

(3)非独裁性: $\wedge_{i \in N} \diamond \neg(\sigma \leftrightarrow i)$ 。

对于偏好聚合逻辑 $J A L\left(\mathrm{~L}^{K}\right)$ 来说, 任意一个判断聚合规 则 $f$, 都存在一个社会福利函数 $F$, 使得 $f=f^{F}$, 再由 $J A L(\mathrm{~L})$ 的可靠性与完全性, 可得 $J A L\left(\mathrm{~L}^{K}\right)$ 是可靠且完全的:

定理 如果备选方案集 $K$ 是有穷的, 那么对于任一公式 $\Psi \in \mathcal{L}\left(N, A^{K}\right)$, 都有 ${ }_{J A L\left(\mathrm{~L}^{K}\right)} \Psi$ 当且仅当神 ${ }^{s w f} \Psi$ 。

\section{5. 总结与展望}

基于判断聚合逻辑 $J A L$, 我们探究了偏好聚合与判断聚 合之间的关系。JAL的语言可以直接用判断聚合规则进行解
释, 这个逻辑能够表达一些聚合规则, 如多数投票聚合规则 等; 这个逻辑也能够表达一些聚合规则的性质, 如独立性、 非独裁性等。这是一个可靠且完全的公理化系统。这一逻 辑不仅可以用于分析判断聚合问题, 而且还可以作为一个 一般框架来分析包括偏好聚合在内的聚合问题, 从而表明 可以把偏好聚合看作是判断聚合的一个特例, 显现了判断 聚合模型的一般性。

判断聚合的一些不可能性结论已得到不断证明 $([10,11])$, 而其中的重要意义并不在于仅仅证明了不存在令 人满意的聚合规则, 而在于可以让我们深刻洞察到为找到 让人接受的聚合规则, 我们需要放宽哪些聚合要求。在下 一步的研究工作中, 我们将从逻辑的视角探寻判断聚合难 题的一些化解方案。

\section{参考文献(References)}

[1] Thomas Ågotnes, Wiebe van der Hoek and Michael Wooldridge. Towards a logic of social welfare. The 7th Conference on Logic and the Foundations of Game and Decision Theory (LOFT 06).

[2] Thomas Ågotnes, Wiebe van der Hoek and Michael Wooldridge. On the logic of preference and judgment aggregation. Autonomous Agents and Multi-Agent Systems (2011)22: 4-30.

[3] K. J. Arrow. Social Choice and Individual Values. New York: Wiley, 1951; 2d edition, 1963.

[4] P. Blackburn, M. de Rijke and Y. Venema. Modal Logic. Cambridge University Press, 2001.

[5] P. Blackburn, J. van Benthem, F. Wolter Ed. Handbook of Modal Logic. Amsterdam: Elsevier, 2007.

[6] Dietrich, F. and List, C. Arrow's theorem in judgement aggregation. Social Choice and Welfare,2007,29 (1),19-33.

[7] Ehlers, L. and Storcken, T. Arrow's possibility theorem for one dimensional single-peaked preferences. Games and Economic Behavior,2008,64(2),533-547.

[8] W. Gaertner. A Primer in Social Choice Theory. New York: Oxford University Press, 2009.

[9] Davide Grossi. Correspondences in the Theory of Aggregation. Lecture Notes in Computer Science, 2010, Volume 6006, Logic and the Foundations of Game and Decision Theory - LOFT 8, Pages 34-60.

[10] List, C. The theory of Judgment aggregation:An introductory review. Synthese,2012,187(1),179-207.

[11] List, C. and Puppe, C. Judgment aggregation:a survey. In Anand, P.,Puppe, C., Pattanaik, P.(eds.), Oxford Handbook of Rational and Social Choice, New York: Oxford University Press, 2009. 